Sādhanā Vol. 28, Part 6, December 2003, pp. 1011-1018. ( ) Printed in India

\title{
Effect of deformation and dielectric filling on electromagnetic propagation through waveguides
}

\author{
AJAY CHAUDHARI \\ Department of Physics, Dr B A M University, Aurangabad 431 004, India \\ Present address: Dept. of Chemistry, National Chung-Cheng University, \\ Ming-Hsiung, Chia-Yi- 621, Taiwan \\ e-mail: ajaychau5@yahoo.com
}

MS received 19 August 2001; revised 2 November 2002

\begin{abstract}
The effect of depression and protrusion of vertical walls and dielectric filling on electromagnetic propagation through $x$-band rectangular waveguides is studied using the finite element method. The effect of these deformations and dielectric filling on $\mathrm{TE}_{10}, \mathrm{TE}_{20}$ mode cutoff frequencies and passband is studied. The results are compared with those of $x$-band rectangular waveguides with depression and protrusion with air medium. $\mathrm{TE}_{10}, \mathrm{TE}_{20}$ mode cutoff frequencies for waveguides with depression and protrusion and filled with dielectric medium are less than that for air medium. However, the bandwidth in some cases of dielectric medium is larger than that for air medium.
\end{abstract}

Keywords. Waveguides; finite element method; depression; protrusion; dielectric filling; cutoff frequencies.

\section{Introduction}

The study of electromagnetic propagation through various guiding structures and devices is of great significance in the development of microwave communication networks and technology. However, in the physical development of the guiding structures, there is always a possibility of the occurrence of some irregularities in these guiding structures. Many workers (Daly 1971; Ikeuchi et al 1981; Mabaya et al 1981) have used the vectorial finite element method (FEM) in terms of longitudinal electric field $\left(E_{z}\right)$ and magnetic field $\left(H_{z}\right)$ to compute the mode spectrum of the waveguide accurately, with arbitrary cross-section. The most serious difficulty in using FEM analysis for waveguides is the appearance of so-called spurious, nonphysical modes (Daly 1971; Ikeuchi et al 1981; Mabaya et al 1981; Hayata et al 1986, 1989).

The vectorial FEM in terms of all three components $\left(H_{x}, H_{y}, H_{z}\right)$ of the magnetic field is proposed by Konard (1977) in which spurious solutions do appear (Rahman \& Davies 1984; Koshiba et al 1989). Davies et al (1982) noted that the spurious solutions in the three component formulation do not satisfy the divergence relation for $\mathbf{H}$, i.e. $\nabla . \mathbf{H}=0$. Koshiba et al (1985) has suggested an improved FEM for analysis of dielectric waveguides in terms of all three components of $\mathbf{H}$. Waveguides with arbitrary shapes are analysed by Bulley \& 
Davies (1969) using the Rayleigh-Ritz method. Dispersion characteristics of arbitrary-shaped waveguides with sharp metal edges are determined by Webb (1988). Srba et al (1994) have used mediam vectorial FEM for analysing rectangular waveguides half filled with dielectric media. Rectangular dielectric waveguide structures are analysed by Bierwirth et al (1986) using the finite difference method. Dielectric strip inserted waveguide is analysed by Chaudhari et al (1998). Finite difference method has been used by Schweig \& Bridges (1984) for the analysis of dielectric waveguides. Rectangular waveguides with deformations are studied by Chaudhari \& Patil $(1996,1997)$.

All the above studies are on waveguides which are either partially filled by dielectric, slab loaded or of arbitrary shape, with air as a dielectric. We have studied the effect of the depression and protrusion of the vertical wall of the waveguide with air medium, on the cutoff frequencies for $\mathrm{TE}_{10}$ and $\mathrm{TE}_{20}$ modes in our previous work (Chaudhari \& Patil 1996). The aim of this paper is to study propagation through $x$-band rectangular waveguides with depression and protrusion of the vertical side, as well as with dielectric filling, and to compare these results with those of waveguides with depression and protrusion, and filled with air. Finite element method with variational principle is the appropriate choice to deal with the irregular geometries of waveguides. The $\mathrm{TE}_{10}$ and $\mathrm{TE}_{20}$ modes are worked out by considering the propagation through waveguides as an eigenvalue problem.

Consider an $x$-band rectangular waveguide with depression and protrusion of the vertical wall. Let the walls of the waveguide be perfectly conducting. The waveguide is filled with dielectric material of dielectric constant 2.5. The cross-section of the waveguide in the $X-Y$ plane of the Cartesian coordinate system is considered a problem domain $\Omega$, with deformed vertical boundaries.

The five cases of deformation considered are: depression on one side, depression on both sides, protrusion on one side, protrusion on both sides, depression on one side and protrusion on other side. For each case of deformation, for different deformation length $L$ in steps of $0.15 \mathrm{~cm}$, the eigenvalues and eigenvectors are obtained. The modes $\mathrm{TE}_{10}$ and $\mathrm{TE}_{20}$ are identified by using the field plots.

\section{Variational formulation}

The electric and magnetic fields inside the waveguide satisfy Maxwell's equations. Thus the problem of electromagnetic propagation under consideration involves solving the Maxwell equations with suitable boundary conditions at the conducting boundaries, such that the tangential component of the electric field is zero on the conductor boundaries.

To avoid spurious solutions, a magnetic field vector formulation is used for the solution of the problem. The details of the formulation can be found in the work by Hayata et al (1986).

The expression for the functional $\Pi$ is

$$
\Pi=\frac{1}{2} \int_{\Omega}\left[\left(\nabla \times H^{*}\right) \cdot\left(\varepsilon^{-1} \nabla \times H\right)-K^{2} H^{*} \cdot H+\left(\nabla \cdot H^{*}\right) \cdot(\nabla \cdot H)\right] \mathrm{d} \Omega .
$$

The stationary character of $\Pi$ requires $\delta \Pi=0$.

The first variation in $\Pi$ is given by

$$
\delta\left[\int_{\Omega}\left[\left(\nabla \times H^{*}\right) \cdot\left(\varepsilon^{-1} \nabla \times H\right)-K^{2} H^{*} H+\left(\nabla \cdot H^{*}\right)(\nabla \cdot H)\right] \mathrm{d} \Omega\right]=0 .
$$


The domain, i.e. cross-section of the waveguide, is divided into rectangular elements with four nodes [Reddy 1996; Akin 1988). The functional over each element is,

$$
\begin{aligned}
\Pi^{e}= & \frac{1}{2}\left[\int_{\Omega^{e}}\left(\nabla \times H^{e^{*}}\right) \cdot\left(\varepsilon^{-1} \nabla \times H^{e}\right) \mathrm{d} \Omega^{e}-\int_{\Omega^{e}} K^{2} H^{e^{*}} \cdot H^{e} \mathrm{~d} \Omega^{e}\right. \\
& \left.+\int_{\Omega^{e}}\left(\nabla \cdot H^{e^{*}}\right) \cdot\left(\nabla \cdot H^{e}\right) \mathrm{d} \Omega^{e}\right],
\end{aligned}
$$

where the unknown vector $\mathbf{H}^{e}$ has three components $\mathbf{H} x, \mathbf{H} y$ and $\mathbf{H} z$. Each unknown component $\mathbf{H} x, \mathbf{H} y$ and $\mathbf{H} z$ is defined in terms of the nodal values $\mathbf{H}^{e} x, \mathbf{H}^{e} y$ and $\mathbf{H}^{e} z$ respectively.

A linear mapping function is used, which needs four nodes per rectangular element.

Functional $\Pi^{e}$ can be written as,

$$
\Pi^{e}=(1 / 2)\left[\left\{\mathbf{H}^{e}\right\}^{T}\left[S^{e}\right]\left\{\mathbf{H}^{e}\right\}-K^{2}\left\{\mathbf{H}^{e}\right\}^{T}\left[T^{e}\right]\left\{\mathbf{H}^{e}\right\}\right] .
$$

The functional for the whole region $\Omega$ is given by

$$
\Pi=(1 / 2)\left[\{\mathbf{H}\}^{T}[S]\{\mathbf{H}\}-K^{2}\{\mathbf{H}\} T[T]\{\mathbf{H}\}\right] .
$$

The condition $\partial \Pi / \partial\{\mathbf{H}\}=0$ leads to the following matrix equation

$$
[S]\{\mathbf{H}\}-K^{2}[T]\{\mathbf{H}\}=0 .
$$

Equation (6) is the matrix equation to be solved for eigenvalues, $K^{2}=\lambda$ and eigenvectors, i.e. Hfield components.

\section{Numerical calculations}

The dimensions of the $x$-band waveguide considered here are $a=2.4 \mathrm{~cm}$ and $b=1.2 \mathrm{~cm}$. For deformation, the corner points and the broad wall $a$ are kept fixed, while the depression and protrusion of the $b$ wall are considered. For the first case of deformation, the depression $L$ from one side is increased in steps of $0.15 \mathrm{~cm}$. For the second case, the depression $L$ is increased from both sides in steps of $0.15 \mathrm{~cm}$ in opposite directions. For the third case, the protrusion $L$ on one side is increased in steps of $0.15 \mathrm{~cm}$. For the fourth case, the protrusion $L$ is increased from both sides in steps of $0.15 \mathrm{~cm}$ in opposite directions. Finally, in the fifth case, the depression $L$ from one side and protrusion $L$ from another side are increased in steps of $0.15 \mathrm{~cm}$.

The cross-section of this waveguide is divided into 128 rectangular elements with four nodes per element. The total number of nodes in the geometry are 153, out of which 48 are the boundary nodes, on which boundary conditions are specified. For each node, there are three unknown field components. Therefore, the matrix problem to be solved for the system, consists of matrices of the order $459 \times 459$. As these matrices are symmetric, only half matrices are stored in the memory. Skyline storage has been used for storing these matrices. The subspace iteration method (Bathe \& Wilson 1987) has been used to solve this eigenvalue problem, and consists of the following three steps. 
Table 1. Bandwidths and cutoff frequencies for $\mathrm{TE}_{10}$ and $\mathrm{TE}_{20}$ mode in gigahertz for waveguides with depressions on one side $(\varepsilon=2 \cdot 5)$.

\begin{tabular}{|c|c|c|c|}
\hline$L(\mathrm{~cm})$ & $\mathrm{TE}_{10}$ & $\mathrm{TE}_{20}$ & Bandwidth \\
\hline 0 & $6 \cdot 2507$ & $12 \cdot 5645$ & $6 \cdot 3138$ \\
\hline $0 \cdot 15$ & $6 \cdot 4744$ & 12.9978 & $6 \cdot 5234$ \\
\hline $0 \cdot 30$ & $6 \cdot 7351$ & $13 \cdot 4718$ & 6.7367 \\
\hline 0.45 & $7 \cdot 0522$ & $13 \cdot 9302$ & $6 \cdot 8780$ \\
\hline $0 \cdot 60$ & 7.4343 & $14 \cdot 8196$ & $7 \cdot 3853$ \\
\hline 0.75 & $7 \cdot 8889$ & $15 \cdot 3966$ & $7 \cdot 5077$ \\
\hline 0.90 & 8.4229 & $16 \cdot 4282$ & 8.0053 \\
\hline 1.05 & 9.0417 & $17 \cdot 7473$ & 8.7055 \\
\hline $1 \cdot 20$ & 9.7466 & 18.7569 & 9.0103 \\
\hline $1 \cdot 35$ & $10 \cdot 5267$ & 19.4945 & $8 \cdot 9678$ \\
\hline
\end{tabular}

(1) Establishment of $q$ starting iteration vectors, $q>p$, where $p$ is the number of eigenvalues and vectors to be calculated.

(2) Use of simultaneous inverse iteration on the $q$ vectors and Ritz analysis to extract the best eigenvalue and eigenvector approximations from the $q$ iteration vectors.

(3) Use of the strum sequence check to verify whether any eigenvalues are missed in the set that is calculated.

For each case, for each $L$, the eigenvalues and eigenvectors are worked out. The eigenvalues obtained are the values of $\omega^{2} \mu_{0} \varepsilon_{0}$. Using these eigenvalues, corresponding cut-off frequencies $f$ and bandwidth are calculated. For each case, for different $L$, the values of $f$ for $\mathrm{TE}_{10}$ and $\mathrm{TE}_{20}$ mode and bandwidth are given in tables $1-5$. The variation of bandwidth with $L$ for the five cases is shown in figures $1-5$.

\section{Results}

For waveguides with depression on one side, depressions on both side and depressions on one side and protrusion on the other, the cutoff frequencies $(f)$ for $\mathrm{TE}_{10}, \mathrm{TE}_{20}$ modes as

Table 2. Bandwidths and cutoff frequencies for $\mathrm{TE}_{10}$ and $\mathrm{TE}_{20}$ mode in gigahertz for waveguides with depressions on one sides $(\varepsilon=2 \cdot 5)$.

\begin{tabular}{|c|c|c|c|}
\hline$L(\mathrm{~cm})$ & $\mathrm{TE}_{10}$ & $\mathrm{TE}_{20}$ & Bandwidth \\
\hline $0 \cdot 0$ & $6 \cdot 2507$ & $12 \cdot 5645$ & $6 \cdot 3138$ \\
\hline $0 \cdot 15$ & $6 \cdot 7042$ & 13.4549 & $6 \cdot 7506$ \\
\hline $0 \cdot 30$ & $7 \cdot 2840$ & $14 \cdot 1972$ & $6 \cdot 9132$ \\
\hline 0.45 & 8.0487 & 15.9908 & 7.9421 \\
\hline 0.60 & $9 \cdot 0512$ & $17 \cdot 5050$ & 8.4538 \\
\hline 0.75 & $10 \cdot 3064$ & 18.3989 & 8.0925 \\
\hline 0.90 & 12.9155 & $20 \cdot 2532$ & $7 \cdot 3377$ \\
\hline 1.05 & $14 \cdot 1502$ & $21 \cdot 3168$ & $7 \cdot 1666$ \\
\hline
\end{tabular}


Table 3. Bandwidths and cutoff frequencies for $\mathrm{TE}_{10}$ and $\mathrm{TE}_{20}$ mode in gigahertz for waveguides with protrusions on one side $(\varepsilon=2 \cdot 5)$.

\begin{tabular}{|c|c|c|c|}
\hline$L(\mathrm{~cm})$ & $\mathrm{TE}_{10}$ & $\mathrm{TE}_{20}$ & Bandwidth \\
\hline 0 & $6 \cdot 2507$ & $12 \cdot 5645$ & $6 \cdot 3138$ \\
\hline $0 \cdot 15$ & $6 \cdot 0814$ & $12 \cdot 0820$ & $6 \cdot 0006$ \\
\hline $0 \cdot 30$ & 5.9384 & 11.9004 & 5.9619 \\
\hline 0.45 & $5 \cdot 8280$ & 11.6460 & $5 \cdot 8180$ \\
\hline $0 \cdot 60$ & $5 \cdot 7455$ & 11.4427 & $5 \cdot 6972$ \\
\hline 0.75 & $5 \cdot 6849$ & $11 \cdot 2814$ & $5 \cdot 5965$ \\
\hline 0.90 & $5 \cdot 6416$ & $10 \cdot 9745$ & $5 \cdot 3328$ \\
\hline 1.05 & $5 \cdot 6117$ & 11.0598 & $5 \cdot 4481$ \\
\hline $1 \cdot 20$ & $5 \cdot 5919$ & $11 \cdot 1925$ & $5 \cdot 6006$ \\
\hline $1 \cdot 35$ & $5 \cdot 5805$ & $11 \cdot 2476$ & $5 \cdot 6671$ \\
\hline
\end{tabular}

Table 4. Bandwidths and cutoff frequencies for $\mathrm{TE}_{10}$ and $\mathrm{TE}_{20}$ mode in gigahertz for waveguides with protrusions on one sides $(\varepsilon=2 \cdot 5)$.

\begin{tabular}{lccc}
\hline$L(\mathrm{~cm})$ & $\mathrm{TE}_{10}$ & $\mathrm{TE}_{20}$ & Bandwidth \\
\hline 0 & $6 \cdot 2507$ & $12 \cdot 5645$ & $6 \cdot 3138$ \\
$0 \cdot 15$ & $5 \cdot 9136$ & $11 \cdot 8668$ & $5 \cdot 9532$ \\
$0 \cdot 30$ & $5 \cdot 6495$ & $11 \cdot 2990$ & $5 \cdot 6495$ \\
$0 \cdot 45$ & $5 \cdot 4537$ & $10 \cdot 8770$ & $5 \cdot 4233$ \\
$0 \cdot 60$ & $5 \cdot 3114$ & $10 \cdot 6896$ & $5 \cdot 3781$ \\
$0 \cdot 75$ & $5 \cdot 2098$ & $10 \cdot 5734$ & $5 \cdot 3635$ \\
0.90 & $5 \cdot 2024$ & $10 \cdot 5577$ & $5 \cdot 3554$ \\
1.05 & $5 \cdot 1327$ & $10 \cdot 4811$ & $5 \cdot 3484$ \\
1.20 & $5 \cdot 0551$ & $10 \cdot 3953$ & $5 \cdot 3403$ \\
1.35 & $5 \cdot 0347$ & $10 \cdot 3798$ & $5 \cdot 3450$ \\
\hline
\end{tabular}

Table 5. Bandwidths and cutoff frequencies for $\mathrm{TE}_{10}$ and $\mathrm{TE}_{20}$ mode in gigahertz for waveguides with depressions on one side and protrusions on other side $(\varepsilon=2 \cdot 5)$.

\begin{tabular}{|c|c|c|c|}
\hline$L(\mathrm{~cm})$ & $\mathrm{TE}_{10}$ & $\mathrm{TE}_{20}$ & Bandwidth \\
\hline 0 & $6 \cdot 2507$ & $12 \cdot 5645$ & $6 \cdot 3138$ \\
\hline $0 \cdot 15$ & $6 \cdot 2845$ & $12 \cdot 6069$ & $6 \cdot 3224$ \\
\hline $0 \cdot 30$ & $6 \cdot 3665$ & $12 \cdot 7008$ & $6 \cdot 3345$ \\
\hline 0.45 & $6 \cdot 5128$ & $12 \cdot 8704$ & $6 \cdot 3576$ \\
\hline $0 \cdot 60$ & 6.7277 & $13 \cdot 2089$ & $6 \cdot 4812$ \\
\hline 0.75 & $7 \cdot 0115$ & $13 \cdot 6171$ & $6 \cdot 6056$ \\
\hline 0.90 & $7 \cdot 3653$ & 14.9462 & $7 \cdot 5809$ \\
\hline 1.05 & 7.7909 & $15 \cdot 1181$ & $7 \cdot 3274$ \\
\hline $1 \cdot 20$ & $8 \cdot 2901$ & $15 \cdot 5089$ & $7 \cdot 2189$ \\
\hline $1 \cdot 35$ & 8.8631 & $16 \cdot 0563$ & $7 \cdot 1932$ \\
\hline
\end{tabular}



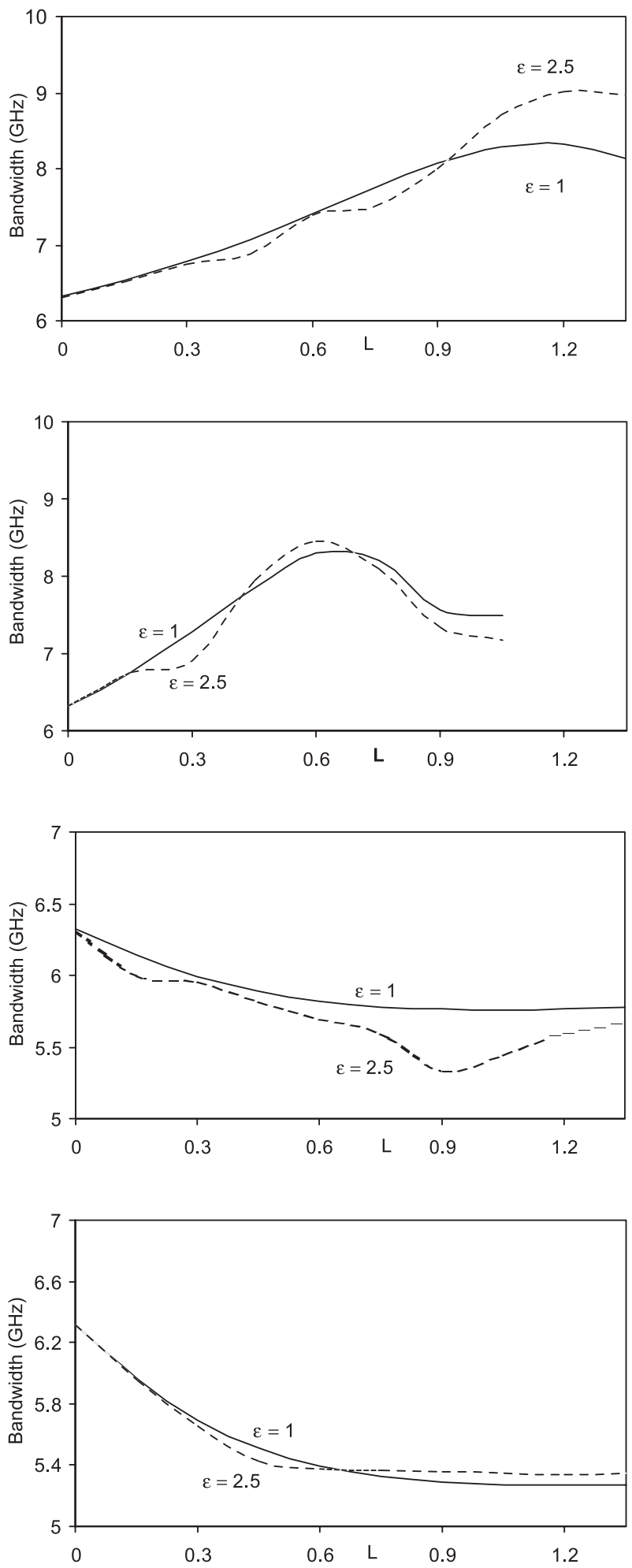

Figure 1. Bandwidths for air and dielectric media for waveguides with depressions on one side.

Figure 2. Bandwidths for air and dielectric media for waveguides with depressions on both sides.

Figure 3. Bandwidths for air and dielectric media for waveguides with protrusions on one side.

Figure 4. Bandwidths for air and dielectric media for waveguides with protrusions on both sides. 


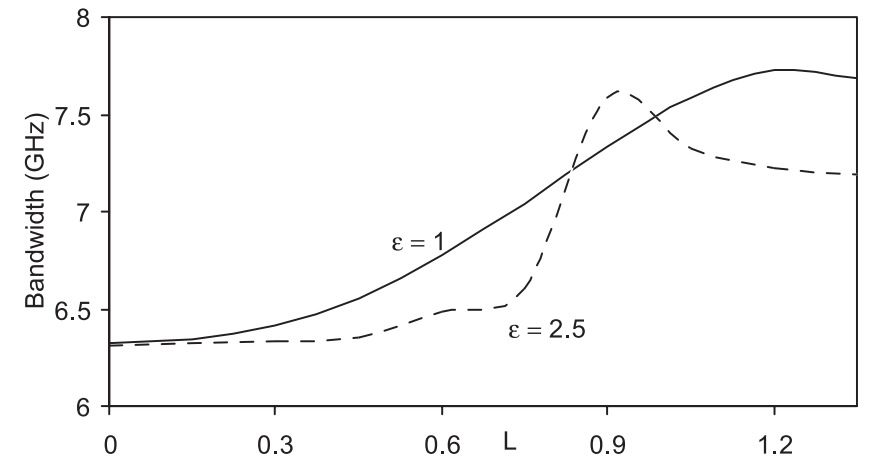

Figure 5. Bandwidths for air and dielectric media for waveguides with depressions on one side and protrusion on the other.

well as bandwidth, increase with deformation length $L$ compared to those for normal $x$ band rectangular waveguides, for waveguides with air as well as dielectric media. However, for waveguides with protrusions on one side or on both sides, these modes decrease with $L$ compared to normal $x$-band rectangular waveguides.

In the case of waveguides with depressions on one side, $f$ for $\mathrm{TE}_{10}, \mathrm{TE}_{20}$ are lower for dielectric medium than for air medium. This decrease in $f$ for $\mathrm{TE}_{20}$ mode is very small but for $\mathrm{TE}_{10}$ mode, the decrease increases with $L$. Due to this, the bandwidth for the lower value of $L$, for the dielectric medium, is almost the same as that for the air medium. But as decrease in $f$ for $\mathrm{TE}_{10}$ mode for higher value of $L$ increases, the bandwidth in this region for the dielectric medium is larger than that for air medium.

For waveguides with depressions on both sides, as the decrease in $f$ for $\mathrm{TE}_{10}, \mathrm{TE}_{20}$ modes for dielectric medium is less than for air medium, the bandwidth is almost same for both the media except in the middle region (for $L 0.45,0.6$ and 0.75 ). In this region, $f$ for $\mathrm{TE}_{20}$ mode is almost the same for both the media but $f$ for $\mathrm{TE}_{10}$ mode, for dielectric medium, is less than that for air medium which causes greater increase in bandwidth for the former.

In the case of waveguides with protrusion on one side, $f$ for both, $\mathrm{TE}_{10}, \mathrm{TE}_{20}$ mode, for dielectric medium, are much lower than that for air medium resulting in greater decrease in bandwidth for dielectric medium than air medium.

For waveguides with protrusions on both side, the decrease in $f$ for $\mathrm{TE}_{10}$ mode for dielectric medium compared to air, is greater after $L=0.6$ (i.e. $L=b / 2$ ) but the decrease in $f$ for $\mathrm{TE}_{20}$ mode is not much. Due to this, the bandwidth for dielectric medium up to the length $L=0.6 \mathrm{~cm}$, is less than that for air medium after which it is greater than for the air medium.

In the case of waveguides with depression on one side and protrusion on the other, $f$ for the $\mathrm{TE}_{10}$ mode decreases more for the dielectric medium than for air but the decrease in $f$ for $\mathrm{TE}_{20}$ mode is not much for lower $L$ values. However at higher $L$ value, the decrease is more, and causes greater decrease in bandwidth for the dielectric medium in this region than for air.

\section{Conclusion}

$x$-Band rectangular waveguides with depressions and protrusions of the vertical sides have been analysed using Finite Element Method for dielectric medium. These results are compared with those for air. The cutoff frequencies for $\mathrm{TE}_{10}, \mathrm{TE}_{20}$ modes decrease for dielectric medium compared to those for air. However this decrease is not the same for both the modes. Due to this the bandwidth is some cases for dielectric medium is larger than that for air medium. 


\section{References}

Akin J E 1988 Application and implementation of finite element method (New York: Academic Press) Bathe K J, Wilson E L 1987 Numerical methods in finite element analysis (New Delhi: Prentice Hall) Bierwirth K, Schulz N, Arndt F 1986 Finite difference analysis of rectangular waveguide structure. IEEE Trans. Microwave Theory Tech. 34: 1104-1114

Bulley R M, Davies J B 1969 Comparison of approximate polynomial solutions to TE modes in an arbitrary shaped waveguide. IEEE Trans. Microwave Theory Tech. 17: 440-447

Chaudhari A, Karkare M, Patil P B 1998 Finite element analysis of dielectric strip inserted waveguide. Indian J. Pure Appl. Phys. 36: 97-100

Chaudhari A S, Patil P B 1996 Finite element analysis of rectangular waveguide with deformation as depression and protrusion of side walls. Indian J. Phys. B70: 475-485

Chaudhari A S, Patil P B 1997 Analysis of shape deformed waveguide using Finite element method. Indian J. Pure Appl. Phys. 35: 57-61

Daly P 1971 Hybrid mode analysis of microstrip by finite element method. IEEE Trans. Microwave Theory Tech. 19: 19-26

Davies J B, Fernandez F A, Phillipou G V 1982 Finite element analysis of all modes in cavities with circular symmetry. IEEE Trans. Microwave Theory Tech. 30: 1975-1982

Hayata K, Koshiba M, Ehuchi M, Suzuki M 1986 Vectorial finite element method without spurious solutions for dielectric waveguide problems using transverse magnetic field components. IEEE Trans. Microwave Theory Tech. 34: 1120-1124

Hayata K, Ehuchi M, Koshiba M 1989 Finite element formulation for guided wave problem using transverse electric field component. IEEE Trans. Microwave Theory Tech. 37: 256-261

Ikeuchi M, Sawami H, Niki H 1981 Analysis of open type dielectric waveguide by finite element iterative method. IEEE Trans. Microwave Theory Tech. 29: 234-239

Konard A 1977 Higher order triangular finite elements for electromagnetic waves in anisotropic media. IEEE Trans. Microwave Theory Tech. 25: 253-259

Koshiba M, Hayata K, Suzuki M 1985 Improved finite element formulation in terms of magnetic field vector for dielectric waveguides. IEEE Trans. Microwave Theory Tech. 33: 227-233

Koshiba M, Hayata K, Suzuki M 1989 Vectorial finite element formulation without spurious solutions for dielectric waveguide. Tech. Res. Rep., Inst. Elect. Commun. Eng. Jpn. 83: 70-85

Mabaya N, Lagasse P E, Vandenbuleke P 1981 Finite element analysis of optical waveguides. IEEE Trans. Microwave Theory Tech. 20: 600-609

Rahman B M A, Davies J B 1984 Finite element analysis and optical waveguide problem. IEEE Trans. Microwave Theory Tech. 32: 20-26

Reddy J N 1986 An introduction to finite element method (New York: McGraw Hill)

Schweig E, Bridges W B 1984 Computer analysis of dielectric waveguide: A finite difference method. IEEE Trans. Microwave Theory Tech. 32: 531-541

Srba R C, Zhao A P, Punjani M 1994 An implementation of the vector finite element analysis of anisotropic waveguide through a general purpose PDE software. IEEE Trans. Microwave Theory Tech. 42: 2311-2318

Webb J P 1988 Finite element analysis of dispersion in waveguides with sharp metal edges. IEEE Trans. Microwave Theory Tech. 36: 1819-1824 